Published in final edited form as:

Radiother Oncol. 2019 July ; 136: 44-49. doi:10.1016/j.radonc.2019.03.024.

\title{
Dose-dependent atrophy of the amygdala after radiotherapy
}

\author{
Minh-Phuong Huynh-Le, MD' ${ }^{1}$, Roshan Karunamuni, $\mathrm{PhD}^{1}$, Vitali Moiseenko, $\mathrm{PhD}^{1}$, \\ Nikdokht Farid, $\mathbf{M D}^{2}$, Carrie R. McDonald, $\mathbf{P h D}^{1,3}$, Jona A. Hattangadi-Gluth, MD ${ }^{1}$, Tyler M. \\ Seibert, MD, PhD ${ }^{1,4}$ \\ ${ }^{1}$ Department of Radiation Medicine and Applied Sciences, University of California San Diego, La \\ Jolla, CA \\ ${ }^{2}$ Department of Radiology, University of California San Diego, La Jolla, CA \\ ${ }^{3}$ Department of Psychiatry, University of California San Diego, La Jolla, CA \\ ${ }^{4}$ Department of Bioengineering, University of California San Diego, La Jolla, CA
}

\section{Abstract}

Background and Purpose: The amygdalae are deep brain nuclei critical to emotional processing and the creation and storage of memory. It is not known whether the amygdalae are affected by brain radiotherapy (RT). We sought to quantify dose-dependent amygdala change one year after brain RT.

Materials and Methods: 52 patients with primary brain tumors were retrospectively identified. Study patients underwent high-resolution, volumetric magnetic resonance imaging before RT and 1 year afterward. Images were processed using FDA-cleared software for automated segmentation of amygdala volume. Tumor, surgical changes, and segmentation errors were manually censored. Mean amygdala RT dose was tested for correlation with amygdala volume change 1 year after RT via the Pearson correlation coefficient. A linear mixed-effects model was constructed to evaluate potential predictors of amygdala volume change, including age, tumor hemisphere, sex, seizure history, and bevacizumab treatment during the study period. As 51 of 52 patients received chemotherapy, possible chemotherapy effects could not be studied. A two-tailed $p$-value $<0.05$ was considered statistically significant.

Results: Mean amygdala RT dose $(r=-0.28, p=0.01)$ was significantly correlated with volume loss. On multivariable analysis, the only significant predictor of amygdala atrophy was radiation

Corresponding Author: Tyler M. Seibert, MD, PhD, Assistant Professor, Department of Radiation Medicine and Applied Sciences, University of California San Diego, 9500 Gilman Dr. Mail Code 0861, La Jolla, CA 92093-0861, tseibert@ ucsd.edu.

Statistical Analyses: Drs. Huynh-Le and Seibert had full access to the data in this study and take responsibility for the integrity of the data and the accuracy of the data analysis.

Conflicts of Interest: Drs. Seibert and Hattangadi-Gluth report grant funding from Varian Medical Systems, unrelated to the present study. Dr. Seibert reports grants from Radiological Society of North America during the conduct of the study and honoraria from WebMD, Inc, for providing educational content.

Publisher's Disclaimer: This is a PDF file of an unedited manuscript that has been accepted for publication. As a service to our customers we are providing this early version of the manuscript. The manuscript will undergo copyediting, typesetting, and review of the resulting proof before it is published in its final citable form. Please note that during the production process errors may be discovered which could affect the content, and all legal disclaimers that apply to the journal pertain. 
dose. The final linear mixed-effects model estimated amygdala volume loss of $0.17 \%$ for every 1 Gy increase in mean amygdala RT dose $(p=0.008)$.

Conclusions: The amygdala demonstrates dose-dependent atrophy one year after radiotherapy for brain tumors. Amygdala atrophy may mediate neuropsychological effects seen after brain RT.

\section{Keywords}

brain radiotherapy; amygdala; quantitative MRI

\section{Introduction}

The amygdalae are medial temporal lobe nuclei critical for processing emotions ${ }^{1-3}$ and both the creation and storage of memory ${ }^{4}$. They have been implicated in cognitive and emotional changes in patients with a wide variety of neurological diseases, including mesial temporal lobe epilepsy, Huntington disease, Alzheimer disease, and frontotemporal dementia ${ }^{5-9}$. In patients with brain tumors, damage to the amygdalae has been associated with precipitation of neuropsychological symptoms, including impaired emotional recognition, anxiety, and depression ${ }^{10,11}$. However, the potential for treatment-induced amygdala damage has not been well studied.

Brain radiotherapy (RT) is standard for patients with brain tumors, but is associated with neuropsychological decline ${ }^{12}$. Up to $50-90 \%$ of brain RT patients who survive $>6$ months after treatment experience some neuropsychological decline, a significant concern for patient quality of life ${ }^{13-16}$. Memory loss is among the most common deficits ${ }^{16}$. Patients with brain tumors also have high rates of depression, stress, and anxiety ${ }^{17-19}$. Efforts to reduce treatment-related toxicity may be improved by identification of neuroanatomical structures that not only are critical to cognitive and emotional function, but also exhibit RT dosedependent damage ${ }^{20,21}$. The amygdala is the primary brain structure responsible for emotional control and processing, and it acts synergistically with the adjacent hippocampus to form memories with emotional context ${ }^{22}$.

In the present study, we aimed to quantify the effects of brain RT on the amygdala using longitudinal, volumetric magnetic resonance (MR) imaging. Previous work with quantitative MRI found RT dose-dependent atrophy of the hippocampus, and recent prospective clinical trial data showed that lower hippocampal RT doses better preserves cognition ${ }^{23-25}$. We hypothesized that the nearby amygdala would also exhibit radiation dose-dependent atrophy, a finding that might justify study of amygdala dysfunction in brain tumor survivors, as well as strategies to reduce negative effects on patient quality of life. Such evidence is important because amygdala damage could have considerable impact on a patient's cognitive and/or emotional functioning, while going poorly appreciated in routine clinical follow-up, where thorough neuropsychological evaluation is often lacking. 


\section{Methods}

\section{Patients}

This study was conducted in accordance with approval by the Institutional Review Board. Fifty-eight adult patients with primary brain tumors treated from 2010-2014 with fractionated, partial-brain radiotherapy (1.8-2.0 Gy per fraction) were retrospectively identified. Doses received ranged from $50.4 \mathrm{~Gy}$ to $60 \mathrm{~Gy}$ (median $60 \mathrm{~Gy}$ ). Inclusion criteria required that patients had standardized MR imaging prior to (or within the first week of) RT start and approximately one year after completion of RT and that patients did not receive prior RT. Six patients were excluded: 5 for poor image quality and 1 for large surgical resection that prevented the automated segmentation from completing. Thus, the final patient cohort comprised 52 patients.

\section{Image Acquisition, Processing, and Amygdalae Delineation}

MRIs were acquired before or within the first week of RT initiation and approximately 1 year (range: 9-15 months) after RT using a standardized protocol at our institution using a 3T Signa Excite HDx system (GE Healthcare, Milwaukee, WI) with an 8-channel head coil, as described previously ${ }^{24}$. Images acquired include 3D volumetric T1-weighted inversion recovery spoiled gradient-echo sequence (TE, $2.8 \mathrm{~ms}$; TR, $6.5 \mathrm{~ms}$; TI, $450 \mathrm{~ms}$ ) obtained preand post- administration of intravenous gadolinium contrast and a 3D T2-weighted Fluid attenuated inversion recovery (FLAIR) sequence (TE, $126 \mathrm{~ms}$; TR, $6000 \mathrm{~ms}$; TI, $1863 \mathrm{~ms}$ ). All images were visually reviewed independently by two physicians for quality control prior to further image processing.

Pre-contrast T1-weighted images were processed using NeuroQuant (CorTechs Labs, Inc, La Jolla, CA), to auto-segment the amygdala. NeuroQuant has received US Food and Drug Administration and Conformite Europeene clearance for clinical use in measuring amygdala volume. Full segmentation is based upon a probabilistic atlas; details have been previously described ${ }^{26}$. Volume measurements (in $\mathrm{mL}$ ) with a color overlay for each amygdala were generated (Figure 1). All color images were visually reviewed by two radiation oncologists for quality assurance of accurate processing. Individual amygdalae were manually censored if the tumor or surgical bed caused poor segmentation.

\section{Amygdala RT Dose Determination}

These auto-segmented pre-contrast $\mathrm{T} 1$-weighted images were then rigidly co-registered to each patient's RT plan (computed tomographic [CT] simulation images); full methodology is described elsewhere ${ }^{24,27}$. This registration was visually confirmed for accuracy and the RT dose from the CT images was resampled into the T1-weighted MRI volume space.

\section{Statistical Analyses}

Analyses were performed using $\mathrm{SciPy}^{28}$, a Python-based mathematical computing software (www.scipy.org), and the R environment for statistical computing ${ }^{29}$. A two-tailed $p$-value of $<0.05$ was considered statistically significant. 
The mean and maximum RT dose to each amygdala were calculated using the registered RT dose data and segmented MRI. For patients receiving other than 30 RT fractions, RT doses were converted at each voxel location in the volume to a 30 -fraction biologically equivalent dose, using an $\alpha / \beta$ for brain tissue of $2 \mathrm{~Gy}$, for direct comparison ${ }^{30,31}$.

Change in amygdala volume was defined as the difference between the volume at 1-year post-RT and the pre-RT volume. Percent change in amygdala volume was calculated as the change in amygdala volume divided by the pre-RT volume for each amygdala. A Pearson correlation coefficient was calculated to determine if a relationship existed between mean (or maximum) dose to an individual amygdala and percent volume change.

As a secondary illustration of effect size, the median and interquartile range (IQR) were calculated for amygdalae receiving relatively higher and lower RT dose. Based on a published breakpoint analysis of radiation dose-related atrophy in cortical gray matter, amygdalae were analyzed depending on whether they received doses $\geq$ or $<34.6 \mathrm{~Gy}^{27}$.

Finally, a linear mixed-effects (LME) model was created using Ime4 version 1.1-7 in R to test for other potential predictors of amygdala volume change. The dependent variable was defined as percent volume change, with a patient-specific random intercept. Mean dose to each individual amygdala was included as the primary fixed effect. Age, hemisphere (left or right), sex, history of one or more major seizures during the study period (generalized tonicclonic or status epilepticus), and history of bevacizumab treatment during the study period were added as potential fixed-effects predictors. As 51 of the 52 patients in our cohort received chemotherapy during the study period (50 of whom received concurrent chemoradiotherapy), chemotherapy was not included as a potential predictor in the model. The addition of each of these potential covariates was evaluated in a forward, stepwise fashion using a likelihood ratio test; each covariate was included as a fixed effect in the final model if $p<0.05$. Restricted maximum likelihood was used to estimate the fixed effects parameters in the final model.

\section{Results}

\section{Patients}

The demographics of the 52 patients included in this study are detailed in Table 1. Briefly, the majority of patients were male $(\mathrm{n}=35,67 \%)$, and the median age of patients at the time of treatment was 54 years (range 19-77). Most patients had grade III-IV glioma ( $\mathrm{n}=42$, $81 \%)$. The most common tumor locations were frontal lobe $(\mathrm{n}=18,35 \%)$ and temporal lobe $(\mathrm{n}=16,31 \%)$. All patients were treated with intensity modulated RT, and most patients $(\mathrm{n}=42,81 \%)$ received 30 fractions of partial-brain RT. The vast majority of patients received chemotherapy during the study period $(\mathrm{n}=51,98 \%)$. Fifteen patients $(29 \%)$ had a major seizure during the study period and ten (19\%) patients received bevacizumab.

\section{Amygdala Analysis}

After censoring for effects of tumor, surgery, or segmentation error, 40 right and 44 left amygdalae (total of 84 amygdalae) were included in the analyses. No patient had both amygdalae censored. Mean dose to the amygdala $(r=-0.28, p=0.01)$ was significantly 
correlated with volume loss, Figure 2. Maximum dose to the amygdala $(r=-0.28, p=0.01)$ was also significantly correlated with volume loss. Fourteen amygdalae received $\geq 34.6 \mathrm{~Gy}$. The median volume percent change in these amygdalae was $-5.74 \%$ (IQR: $-16.67,1.84$ ). In the 70 amygdalae that received $<34.6 \mathrm{~Gy}$, the median volume percent change was $1.92 \%$ (IQR: $-3.82,8.15$ ).

Results of linear mixed-effects modeling with RT dose as the main fixed effect and patient (random effect) as predictors of amygdale volume change are presented in Table 2. No other covariates (age, hemisphere, patient sex, seizure history, or bevacizumab history) significantly contributed to the model after likelihood ratio testing ( $>>0.05$ for these covariates). The dose estimate for the final model, -0.17 , corresponds to the rate of amygdala percent volume loss observed for every $1 \mathrm{~Gy}$ increase in mean dose received to the structure $(p=0.008)$.

\section{Discussion}

To our knowledge, this is the first longitudinal evaluation of radiotherapy effects on the amygdala. In adults with primary brain tumors, we found radiation dose-dependent atrophy of the amygdala. On multivariable analysis, only RT dose significantly predicted for amygdala atrophy. The amygdalae are crucial to the processing of emotion and the emotional aspects of memory, and our study suggests that the clinical effects of brain RT on these structures need to be further investigated as they could lead to, or exacerbate, emotion dysregulation in patients with brain tumors.

Changes to the amygdala have been associated with impairment of patient function and cognition in a number of neurological and neuropsychological disorders. In patients with Huntington disease, smaller amygdala volumes were found to be associated with worse cognitive and motor performance in symptomatic patients ${ }^{7}$. Even in pre-symptomatic patients with Huntington disease, smaller amygdalae volumes were associated with increased anxiety levels ${ }^{7}$. In a series of military veterans, those with PTSD had significantly smaller amygdala volume (3.5\% smaller in the left and 5.0\% smaller in the right) than trauma-exposed veterans without PTSD ${ }^{32}$. The amygdala has also been implicated in patients with mesial temporal lobe epilepsy ${ }^{5}$. Additionally, in some patients with temporal lobe epilepsy, the epileptogenic focus involves the amygdala ${ }^{33}$. Seizures are of particular interest in the brain tumor population, as they can be the presenting symptom in $20-40 \%$ of patients, and up to $45 \%$ of these patients can also develop seizures throughout the course of their disease ${ }^{34}$. While we did not find that a history of major seizures during the study period was predictive of amygdala atrophy, larger cohorts may reveal a potential relationship.

The amygdala also plays a role in diseases that affect older adults, including Alzheimer disease. Amygdala atrophy has been correlated with global symptom severity in patients with Alzheimer disease; interestingly, amygdala atrophy was also associated with abnormal motor behavior in these patients, with possible relationships to anxiety and irritability ${ }^{8}$. Moreover, the level of amygdala atrophy in that study was similar to levels of atrophy seen in the hippocampus. Decreased right amygdala volume has also been found to precede the initial clinical signs of cognitive decline in elderly patients ${ }^{6}$. Research evaluating the impact 
of aging on the amygdala has shown that healthy aging does not significantly damage the amygdala structure ${ }^{35-37}$. Similarly, we found that age was not a significant predictor for amygdala volume change in our cohort of brain tumor patients, as opposed to previously described results in the hippocampus ${ }^{24}$. Amygdala atrophy and its effects on patients in any of these neurological contexts could easily go undetected in current routine clinical followup, where specialized cognitive testing is not readily available, making quantitative neuroimaging techniques especially attractive.

Amygdala volumes have also been associated with impaired neuropsychological and social function in cancer patients. In a cross-sectional series of breast cancer survivors, patients who had intrusive recollections (symptoms of post-traumatic stress disorder [PTSD]) had smaller amygdala volumes determined on MRI compared to breast cancer controls without intrusive recollections ${ }^{38}$. Another study evaluating the relationship between amygdala volume and depression in breast cancer survivors revealed that left amygdala volumes were smaller in patients who had experienced a depressive episode ${ }^{39}$. Additionally, high levels of social support in breast cancer survivors have been linked with reduced amygdala reactivity and decreased inflammatory markers ${ }^{40}$.

Given the established association between amygdala atrophy and neuropsychological dysfunction in an array of brain disorders, the present finding of amygdala atrophy related to RT dose has intriguing potential implications for RT planning. Recently published literature evaluating RT effects on the amygdala showed a significant relationship between post-RT amygdala volumes and full-scale IQ and verbal learning memory in a series of children with acute lymphoblastic leukemia (ALL) ${ }^{41}$. In that study, children who received whole-brain RT (WBRT) with chemotherapy had smaller amygdala volumes than those treated with chemotherapy alone, but amygdala volumes were similar in controls (patients with ALL who had not yet started treatment) and children who received chemotherapy. Pre-clinical data also implicate radiotherapy to the amygdala in changes to olfactory memory processing: a study using a mouse model of pediatric radiotherapy revealed significant reductions in diffusion tensor imaging of amygdala volume three months post-WBRT ${ }^{42}$. Cognitively, irradiated male mice had impaired odor-recognition memory in adulthood, compared to nonirradiated males. Altogether, these data suggest that radiation to the amygdala can influence neuropsychological consequences after RT, particularly for memory.

Amygdala RT dose reduction is especially relevant when considering the amygdala's close proximity to-and interactions with—-the hippocampus, a structure that is already being studied for avoidance ${ }^{21}$. Both the amygdala and hippocampus reside in the medial temporal lobe and are critical parts of the limbic system circuitry. The hippocampus and amygdala both contribute to memory systems, with the hippocampus involved in the consolidation of long-term, episodic, or declarative memory (such as conscious fact-recall) and the amygdala more involved in emotional memory and memories with emotional context ${ }^{22,43}$. The amygdala and hippocampus also have neural connections thought to allow them to work in concert for the development and preservation of emotional and remote memory $22,43,44$. Physiologically, the basolateral complex of the amygdala has substantial projections to the memory structures of the medial temporal lobe, with afferents to the hippocampus ${ }^{45,46}$, while the hippocampus has reciprocal projections (mainly originating in the subicular 
region) that send sensory information to the amygdala ${ }^{45,47}$. The amygdala can therefore shape the coding and consolidation of hippocampal-dependent memories, while the hippocampus can create episodic representations of the emotional importance of specific stimuli, thus affecting the amygdala response to emotional events ${ }^{43}$. This connectivity may have implications for memory preservation in brain tumor patients. We have shown here that the amygdala demonstrates similar dose-dependent atrophy after RT to that previously shown in the hippocampus ${ }^{24},(r=-0.28$ for the amygdala and -0.24 for the hippocampus $)$.

Hippocampal avoidance in RT planning is already an active area of clinical research to improve memory preservation. The NRG-CC001 study randomized patients receiving memantine and WBRT to plans with or without hippocampal sparing. Preliminary results were presented recently and showed that patients who received hippocampal-sparing WBRT had greater memory preservation ${ }^{23}$. Of note, hippocampal avoidance in that trial was achieved via a $5 \mathrm{~mm}$ uniform expansion around the hippocampus, implying there was likely considerable dose reduction to the bilateral amygdalae, as well. While amygdala avoidance was not a focus of CC001, it is possible that the favorable dosimetry for the amygdala had some contribution to memory preservation.

It is also important to note that not all areas of the brain have been shown to have radiation dose-dependent atrophy. A prior study of RT-related changes to regions of the cerebral cortex demonstrated dose-dependent atrophy in the entorhinal and inferior parietal cortex (areas associated with memory and executive functioning), but not in the pericalcarine cortex or paracentral lobule (areas associated with vision and somatosensory/motor function) ${ }^{20}$. The radiation dose-dependent amygdala atrophy measured here is consistent with the observation that RT dose-dependent changes may be at least partially specific to areas of the brain associated with cognition ${ }^{20,21,24}$.

Limitations of our study include the retrospective design at a single institution. The majority of patients in our cohort had grade III or IV glioma. Future studies could include more patients with other primary brain tumors (who might benefit even more from cognitivesparing radiotherapy). Though our patient cohort included more men than women, sex did not predict for amygdala atrophy. The potential effects of chemotherapy and concurrent chemotherapy could not be evaluated in this cohort as independent risk factors for amygdala atrophy because nearly all of the patients received chemotherapy as part of their clinical care. As such, we cannot determine whether chemotherapy causes amygdala atrophy or whether it interacts with the observed radiation dose. This is important, as chemotherapy has been suggested to potentiate brain gray matter volume $\operatorname{loss}^{48}$. There is also a potential synergistic effect of chemotherapy and RT on amygdala volumes: children with ALL who received methotrexate alone demonstrated amygdala volume loss, but those who received methotrexate along with brain RT had substantially increased volume decline ${ }^{41}$. We are, additionally, unable to quantify cognitive or emotional changes that may have occurred in these patients, as thorough neuropsychological data are not available. The effects of brain radiotherapy on amygdala function, and its potential role in mediating neuropsychological consequences, need to be evaluated in prospective studies; one is ongoing at our institution. We plan to further evaluate radiation dose-dependent amygdala changes in this study. Finally, radiation damage to the brain can evolve over time. A case report of a patient with 
glioblastoma showed unilateral hippocampal wasting that started shortly after completion of chemoradiation, then stabilized after six months (with follow-up to 20 months ${ }^{49}$. While longitudinal trends for amygdala volume loss have not yet been reported, it is plausible that amygdala volumes can also change over time, and this should be investigated in future work.

In conclusion, we present the first evidence of radiation dose-dependent atrophy of the amygdala in patients with primary brain tumors. This amygdala volume loss may contribute to the neuropsychological sequelae observed in patients after brain RT.

\section{Acknowledgments}

Funding Sources: Supported by the Radiological Society of North America Research \& Education Foundation (RR1554 - TMS); National Institutes of Health (1KL2TR001444, UL1TR000100 - JAH-G); National Cancer Institute and UC San Diego Moores Cancer Center (P30 CA02310029 - JAH-G); and American Cancer Society (ACS-IRG 70-002 - CRM). The content is solely the responsibility of the authors and does not necessarily represent the official views of any of the funding agencies, who had no direct role in designing, conducting, or reporting the study.

\section{References}

1. Patin A, Hurlemann R. Modulating amygdala responses to emotion: Evidence from pharmacological fMRI. Neuropsychologia. 2011;49(4):706-717. doi:10.1016/j.neuropsychologia.2010.10.004 [PubMed: 20933529]

2. Sato W, Kochiyama T, Uono S, et al. Rapid amygdala gamma oscillations in response to fearful facial expressions. Neuropsychologia. 2011;49(4):612-617. doi:10.1016/ j.neuropsychologia.2010.12.025 [PubMed: 21182851]

3. Scott SK, Young AW, Calder AJ, Hellawell DJ, Aggleton JP, Johnson M. Impaired auditory recognition of fear and anger fellowing bilateral amygdala lesions. Nature. 1997;385(6613):254257. doi:10.1038/385254a0 [PubMed: 9000073]

4. Markowitsch HJ, Staniloiu A. Amygdala in action: Relaying biological and social significance to autobiographical memory. Neuropsychologia. 2011;49(4):718-733. doi:10.1016/ j.neuropsychologia.2010.10.007 [PubMed: 20933525]

5. Kim DW, Lee SK, Chung CK, Koh YC, Choe G, Lim SD. Clinical features and pathological characteristics of amygdala enlargement in mesial temporal lobe epilepsy. J Clin Neurosci. 2012;19(4):509-512. doi:10.1016/j.jocn.2011.05.042 [PubMed: 22321366]

6. Zanchi D, Giannakopoulos P, Borgwardt S, Rodriguez C, Haller S. Hippocampal and amygdala gray matter loss in elderly controls with subtle cognitive decline. Front Aging Neurosci. 2017;9(3):50. doi:10.3389/fnagi.2017.00050 [PubMed: 28326035]

7. Ahveninen LM, Stout JC, Georgiou-Karistianis N, Lorenzetti V, Glikmann-Johnston Y. Reduced amygdala volumes are related to motor and cognitive signs in Huntington's disease: The IMAGEHD study. Neuroimage Clin. 2018;18:881-887. doi:10.1016/j.nicl.2018.03.027 [PubMed: 29876272]

8. Poulin SP, Dautoff R, Morris JC, Barrett LF, Dickerson BC. Amygdala atrophy is prominent in early Alzheimer's disease and relates to symptom severity. Psychiatry Res - Neuroimaging. 2011;194(1):7-13. doi:10.1016/j.pscychresns.2011.06.014

9. Barnes J, Whitwell JL, Frost C, Josephs KA, Rossor M, Fox NC. Measurements of the amygdala and hippocampus in pathologically confirmed Alzheimer disease and frontotemporal lobar degeneration. Arch Neurol. 2006;63(10):1434-1439. doi:10.1001/archneur.63.10.1434 [PubMed: 17030660]

10. Assefa D, Haque FN, Wong AH. Case report: Anxiety and fear in a patient with meningioma compressing the left amygdala. Neurocase. 2012;18(2):91-94. doi:10.1080/13554794.2011.556126 [PubMed: 22011126] 
11. Campanella F, Shallice T, Ius T, Fabbro F, Skrap M. Impact of brain tumour location on emotion and personality: A voxel-based lesion-symptom mapping study on mentalization processes. Brain. 2014;137(9):2532-2545. doi:10.1093/brain/awu183 [PubMed: 25027503]

12. Makale MT, McDonald CR, Hattangadi-Gluth JA, Kesari S. Mechanisms of radiotherapyassociated cognitive disability in patients with brain tumours. Nat Rev Neurol. 2016;13(1):52-64. doi :10.1038/nrneurol.2016.185 [PubMed: 27982041]

13. McDuff SGR, Taich ZJ, Lawson JD, et al. Neurocognitive assessment following whole brain radiation therapy and radiosurgery for patients with cerebral metastases. J Neurol Neurosurg Psychiatry. 2013;84(12):1384-1391. doi:10.1136/jnnp-2013-305166 [PubMed: 23715918]

14. Saad S, Wang TJC. Neurocognitive Deficits After Radiation Therapy for Brain Malignancies. Am J Clin Oncol. 2015;38(6):634-640. doi:10.1097/COC.0000000000000158 [PubMed: 25503433]

15. Meyers CA, Brown PD. Role and relevance of neurocognitive assessment in clinical trials of patients with CNS tumors. J Clin Oncol. 2006;24(8):1305-1309. doi:10.1200/JCO.2005.04.6086 [PubMed: 16525186]

16. Greene-Schloesser D, Robbins ME, Peiffer AM, Shaw EG, Wheeler KT, Chan MD. Radiationinduced brain injury: A review. Front Oncol. 2012;2:73. doi:10.3389/fonc.2012.00073 [PubMed: 22833841]

17. Huang J, Zeng C, Xiao J, et al. Association between depression and brain tumor: a systematic review and meta-analysis. Oncotarget. 2017;8(55):94932-94943. doi:10.18632/oncotarget.19843 [PubMed: 29212279]

18. Chochinov HM. Depression in cancer patients. Lancet Oncol. 2001;2(8):499-505. doi:10.1016/ S1470-2045(01)00456-9 [PubMed: 11905726]

19. Keir ST, Guill AB, Carter KE, Friedman HS. Stress and intervention preferences of patients with brain tumors. Support Care Cancer. 2006;14(12):1213-1219. doi:10.1007/s00520-006-0087-9 [PubMed: 16733656]

20. Seibert TM, Karunamuni R, Kaifi S, et al. Cerebral Cortex Regions Selectively Vulnerable to Radiation Dose-Dependent Atrophy. Int J Radiat Oncol Biol Phys. 2017;97(5):910-918. doi:10.1016/j.ijrobp.2017.01.005 [PubMed: 28333012]

21. Gondi V, Pugh SL, Tome WA, et al. Preservation of memory with conformal avoidance of the hippocampal neural stem-cell compartment during whole-brain radiotherapy for brain metastases (RTOG 0933): A phase II multi-institutional trial. J Clin Oncol. 2014;32(34):3810-3816. doi:10.1200/JCO.2014.57.2909 [PubMed: 25349290]

22. Richter-Levin G, Akirav I. Amygdala-Hippocampus Dynamic Interaction in Relation to Memory. Mol Neurobiol. 2000;22(1-3):011-020. doi:10.1385/MN:22:1-3:011

23. Gondi V, Deshmukh S, Brown P, et al. 2018 ASTRO Annual Meeting Late-Breaking Abstract Selection LBA9: Preservation of Neurocognitive Function (NCF) with Conformal Avoidance of the Hippocampus during Whole Brain Radiotherapy (HA-WBRT) for Brain Metastases: Preliminary Results of Phase III Tri.; 2018. doi:10.1016/j.ijrobp.2018.08.047

24. Seibert TM, Karunamuni R, Bartsch H, et al. Radiation Dose-Dependent Hippocampal Atrophy Detected With Longitudinal Volumetric Magnetic Resonance Imaging. Int J Radiat Oncol Biol Phys. 2017;97(2):263-269. doi:10.1016/j.ijrobp.2016.10.035 [PubMed: 28068234]

25. Gondi V, Hermann BP, Mehta MP, Tome WA. Hippocampal dosimetry predicts neurocognitive function impairment after fractionated stereotactic radiotherapy for benign or low-grade adult brain tumors. Int J Radiat Oncol Biol Phys. 2013;85(2):345-354. doi:10.1016/j.ijrobp.2012.11.031

26. Brewer JB, Magda S, Airriess C, Smith ME. Fully-automated quantification of regional brain volumes for improved detection of focal atrophy in Alzheimer disease. Am J Neuroradiol. 2009;30(3):578-580. doi:10.3174/ajnr.A1402 [PubMed: 19112065]

27. Karunamuni R, Bartsch H, White NS, et al. Dose-Dependent Cortical Thinning after Partial Brain Irradiation in High-Grade Glioma. Int J Radiat Oncol Biol Phys. 2016;94(2):297-304. doi:10.1016/j.ijrobp.2015.10.026 [PubMed: 26853338]

28. Oliphant TE. Python for scientific computing. Comput Sci Eng. 2007;9(3):10-20. doi:10.1109/ MCSE.2007.58

29. R Core Team. R: A language and environment for statistical computing. In: Vienna, Austria: R Foundation for Statistical Computing ; 2015. 
30. Bruzzaniti V, Abate A, Pedrini M, Benassi M, Strigari L. IsoBED: A tool for automatic calculation of biologically equivalent fractionation schedules in radiotherapy using IMRT with a simultaneous integrated boost (SIB) technique. J Exp Clin Cancer Res. 2011;30(1):52. doi:10.1186/1756-9966-30-52 [PubMed: 21554675]

31. Fowler JF. The linear-quadratic formula and progress in fractionated radiotherapy. Br J Radiol. 1989;62(740):679-694. doi:10.1259/0007-1285-62-740-679 [PubMed: 2670032]

32. Morey R, Gold A, LaBar K, et al. Amygdala volume changes with posttraumatic stress disorder in a large case-controlled veteran group. Arch Gen Psychiatry. 2012;69(11):1169-1178. doi:10.1001/ archgenpsychiatry.2012.50.Amygdala [PubMed: 23117638]

33. Isokawa-Akesson M, Wilson CL, Babb TL. Structurally stable burst and synchronized firing in human amygdala neurons: Auto- and cross-correlation analyses in temporal lobe epilepsy. Epilepsy Res. 1987;1(1):17-34. doi:10.1016/0920-1211(87)90047-7 [PubMed: 3504380]

34. Maschio M Brain Tumor-Related Epilepsy. Curr Neuropharmacol. 2012;10(2):124-133. doi:10.1016/B978-0-12-804066-9.00061-4 [PubMed: 23204982]

35. Soininen HS, Partanen K, Pitkanen A, et al. Volumetric MRI analysis of the amygdala and the hippocampus in subjects with age-associated memory impairment: Correlation to visual and verbal memory. Neurology. 1994;44(9):1660-1660. doi:10.1212/WNL.44.9.1660 [PubMed: 7936293]

36. Grieve SM, Clark CR, Williams LM, Peduto AJ, Gordon E. Preservation of limbic and paralimbic structures in aging. Hum BrainMapp. 2005;25(4):391-401. doi:10.1002/hbm.20115

37. Good CD, Johnsrude IS, Ashburner J, Henson RNA, Friston KJ, Frackowiak RSJ. A voxel-based morphometric study of ageing in 465 normal adult human brains. Neuroimage. 2001;14(1 I):2136. doi:10.1006/nimg.2001.0786 [PubMed: 11525331]

38. Matsuoka Y, Yamawaki S, Inagaki M, Akechi T, Uchitomi Y. A volumetric study of amygdala in cancer survivors with intrusive recollections. Biol Psychiatry. 2003;54(7):736-743. doi:10.1016/ S0006-3223(02)01907-8 [PubMed: 14512214]

39. Yoshikawa E, Matsuoka Y, Yamasue H, et al. Prefrontal cortex and amygdala volume in first minor or major depressive episode after cancer diagnosis. Biol Psychiatry. 2006;59(8):707-712. doi:10.1016/j.biopsych.2005.08.018 [PubMed: 16213471]

40. Muscatell KA, Eisenberger NI, Dutcher JM, Cole SW, Bower JE. Links between inflammation, amygdala reactivity, and social support in breast cancer survivors. Brain Behav Immun. 2016;53:34-38. doi:10.1016/jbbi.2015.09.008 [PubMed: 26384778]

41. Zając-Spychała O, Pawlak M, Karmelita-Katulska K, et al. Anti-leukemic treatment-induced neurotoxicity in long-term survivors of childhood acute lymphoblastic leukemia: impact of reduced central nervous system radiotherapy and intermediate- to high-dose methotrexate. Leuk Lymphoma. 2018;0(0):1-10. doi:10.1080/10428194.2018.1434879

42. Perez EC, Rodgers SP, Inoue T, Pedersen SE, Leasure JL, Gaber MW. Olfactory Memory Impairment Differs by Sex in a Rodent Model of Pediatric Radiotherapy. Front Behav Neurosci. 2018;12. doi:10.3389/fnbeh.2018.00158

43. Phelps EA. Human emotion and memory: Interactions of the amygdala and hippocampal complex. Curr Opin Neurobiol. 2004;14(2):198-202. doi:10.1016/j.conb.2004.03.015 [PubMed: 15082325]

44. Atucha E, Vukojevic V, Fornari R V, et al. Noradrenergic activation of the basolateral amygdala maintains hippocampus-dependent accuracy of remote memory. Proc Natl Acad Sci. 2017;114(34):201710819. doi:10.1073/pnas.1710819114

45. Sah P, Faber ESL, Lopez de Armentia M, Power J. The Amygdaloid Complex: Anatomy and Physiology. Physiol Rev. 2003;83(3):803-834. doi:10.1152/physrev.00002.2003 [PubMed: 12843409]

46. Petrovich GD, Canteras NS, Swanson LW. Combinatorial amygdalar inputs to hippocampal domains and hypothalamic behavior systems. Brain Res Rev. 2001;38(1-2):247-289. doi:10.1016/ S0165-0173(01)00080-7 [PubMed: 11750934]

47. Canteras NS, Swanson LW. Projections of the ventral subiculum to the amygdala, septum, and hypothalamus: A PHAL anterograde tract- tracing study in the rat. J Comp Neurol. 1992;324(2):180-194. doi:10.1002/cne.903240204 [PubMed: 1430328] 
48. Conroy SK, Ahles TA, West JD, Saykin AJ, McDonald BC. Gray matter reduction associated with systemic chemotherapy for breast cancer: a prospective MRI study. Breast Cancer Res Treat. 2010;123(3):819-828. doi:10.1007/s10549-010-1088-4 [PubMed: 20690040]

49. Seibert TM, Dalia Y, Karunamuni R, et al. Unilateral hippocampal wasting after combinedmodality therapy for glioblastoma. Acta Oncol (Madr). 2018;57(5):688-691.

doi:10.1080/0284186X.2017.1398412 


\section{Highlights}

- The amygdala is critical to emotional processing, fear recognition, and memory.

- Radiation dose-dependent amygdala atrophy was measured in a retrospective cohort of 52 primary brain tumor patients who received fractionated, partialbrain radiotherapy.

- Amygdala atrophy associated with radiation dose may contribute to neuropsychological deficits after brain radiotherapy. 


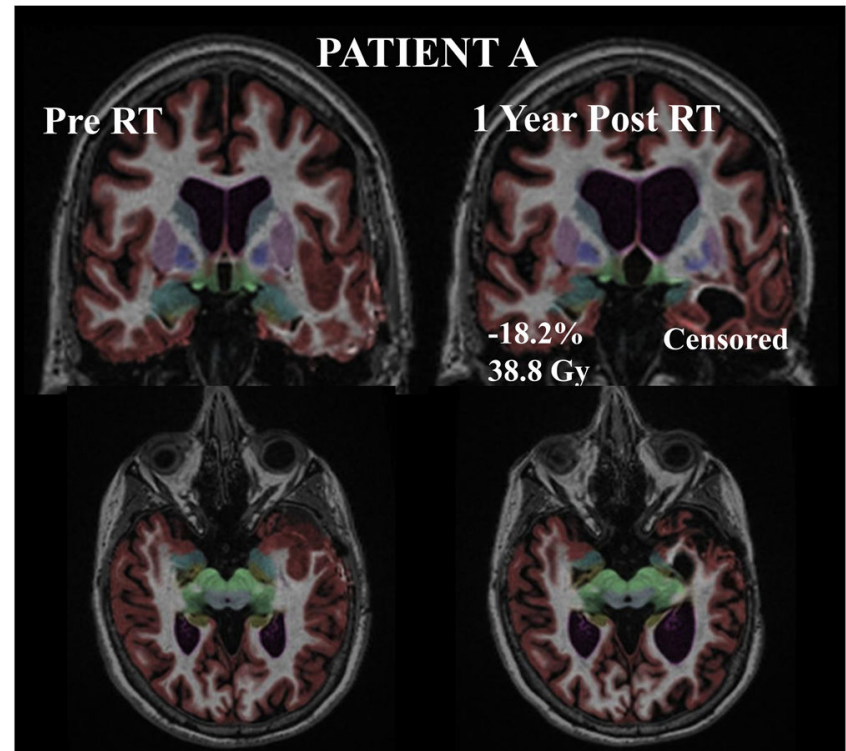

\section{PATIENT B}

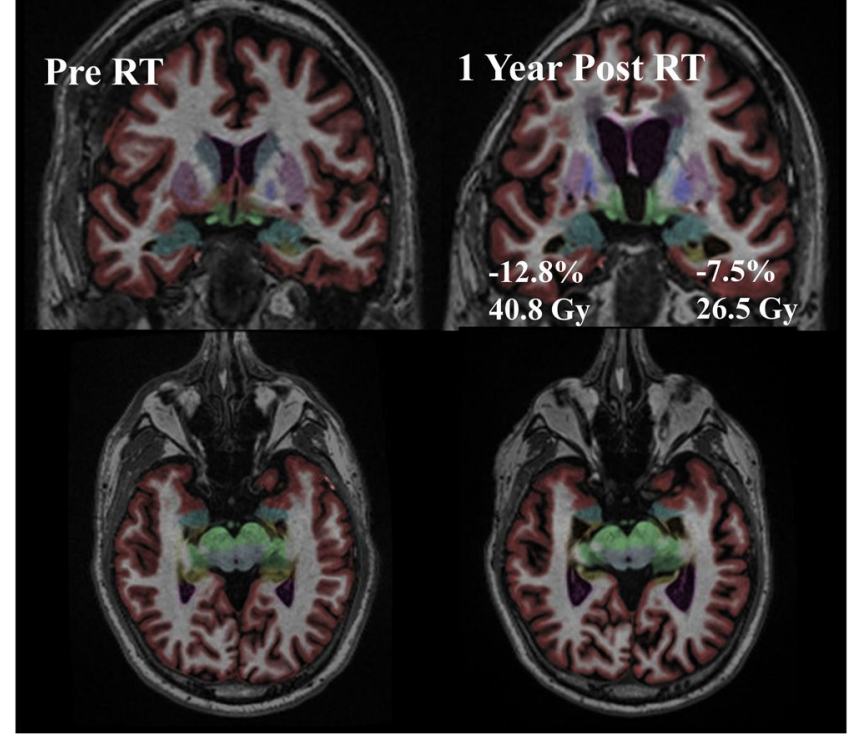

Figure 1.

Magnetic resonance images (MRI) with color overlay from two illustrative cases showing automated segmentation of the amygdala in cyan. Images on left and right are MRIs taken pre-radiotherapy and 1-year post-radiotherapy, respectively. Coronal and axial images are shown for both cases. Volume percent change of the amygdala and mean dose to that structure are shown on the post-radiotherapy coronal MRI slices. 


\section{Radiation dose-dependent Amygdala volume change}

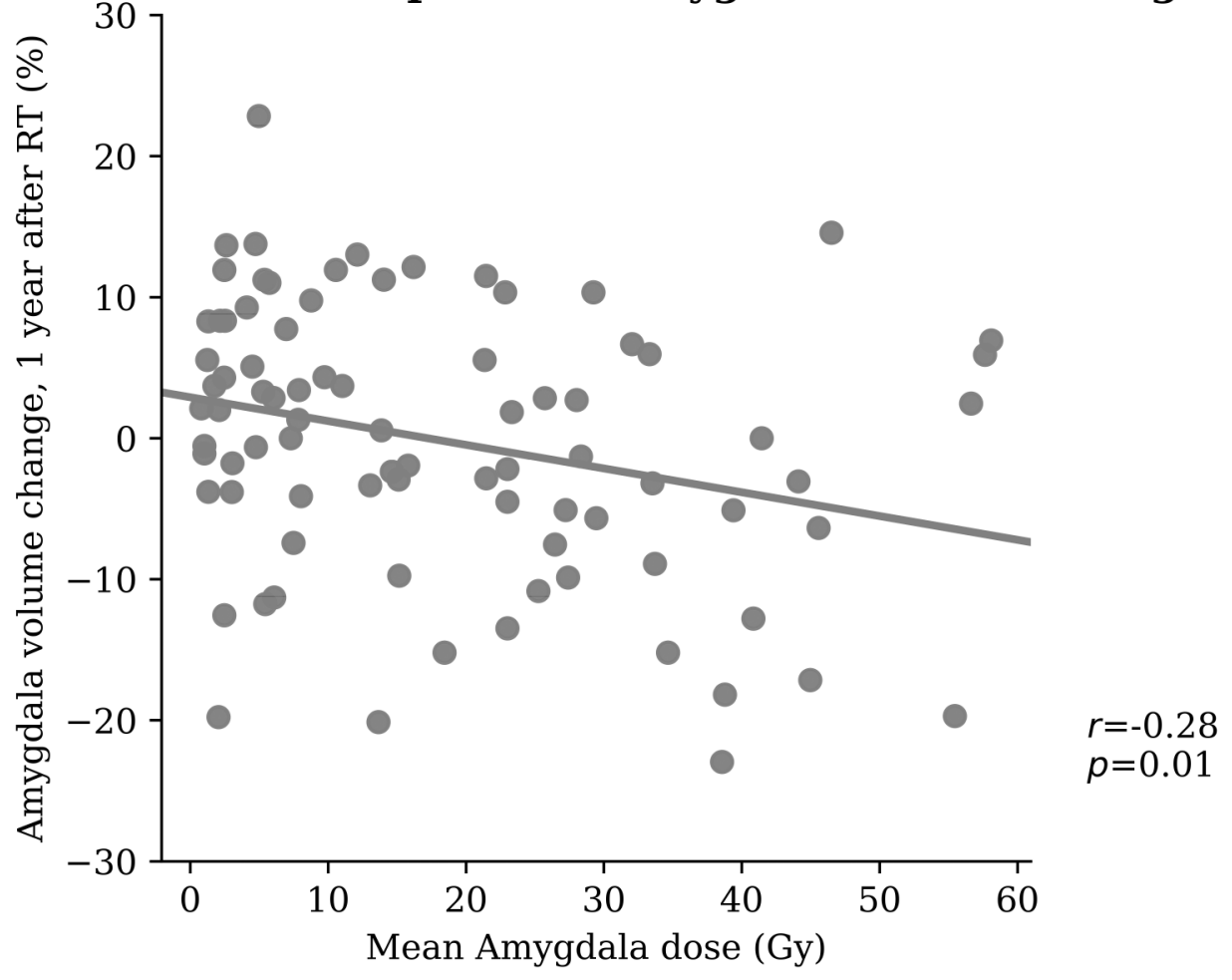

Figure 2.

Amygdala volume changes one year after radiotherapy (RT), reported as percentage change relative to baseline pre-RT volume. Each individual dot corresponds to one amygdala (left or right; 84 amygdalae, in all). 
Table 1.

Demographic and tumor characteristics of the 52 patients included in the cohort.

Characteristic

Age (median in years, range)

Sex

- Male

- Female

Tumor histology

- Grade IV glioma

- Grade III glioma

- Grade II glioma

- Other low-grade glioma

- Low grade glioneural tumor

- Meningioma

Tumor location

- Frontal

- Temporal

- Parietal

- Occipital

- Temporoparietal

- Frontoparietal

- Frontotemporal

- Parietooccipital

- Thalamus

- Cavernous sinus

- Cerebellum

Surgery type

- Gross total resection

- Subtotal resection

- Biopsy

- None*

RT dose, Gy (fraction size)

- 60 (2 Gy)

- 59.4 (1.8 Gy)

- 55.8 (1.8 Gy)

- 54 (1.8 Gy)

- 50.4 (1.8 Gy)

RT technique

- Static intensity modulated RT

- Volumetric modulated arc therapy

Seizure history ${ }^{a}$

- Yes

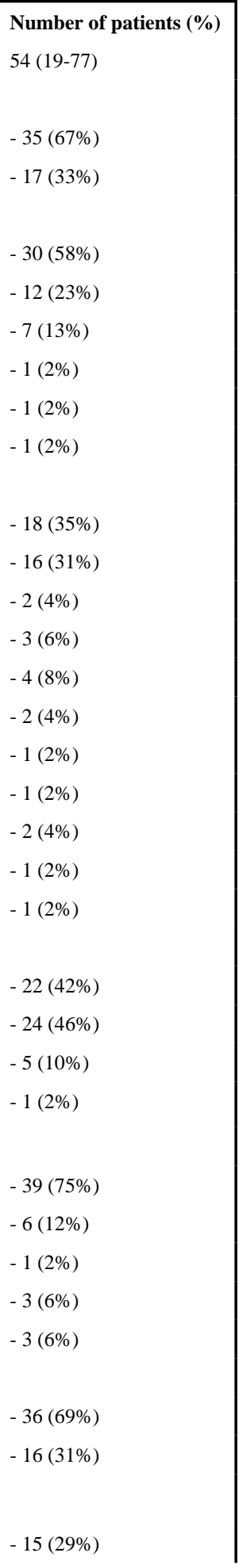




\begin{tabular}{|c|c|c|}
\hline \multirow{10}{*}{ 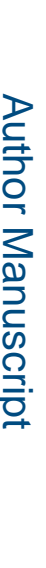 } & Characteristic & Number of patients (\%) \\
\hline & - No & $-37(71 \%)$ \\
\hline & Chemotherapy or other systemic therapy & \\
\hline & - Temozolomide ${ }^{b}$ alone & $-18(35 \%)$ \\
\hline & - Temozolomide ${ }^{b}+$ bevacizumab & $-4(8 \%)$ \\
\hline & - Temozolomide $^{b}+$ bevacizumab + other chemotherapy $c$ & $-5(10 \%)$ \\
\hline & - Temozolomide ${ }^{b}+$ bevacizumab + other clinical trial ${ }^{d}$ & $-1(2 \%)$ \\
\hline & - Temozolomide ${ }^{b}+$ other clinical trial $^{e}$ & $-9(17 \%)$ \\
\hline & - Temozolomide ${ }^{f}+$ other chemotherapy $g$ & $-14(27 \%)$ \\
\hline & - None & $-1(2 \%)$ \\
\hline
\end{tabular}

Tumor diagnosed as meningioma based on imaging characteristics without pathology.

${ }^{a}$ Seizure history defined as patient having a generalized tonic-clonic seizure or status epilepticus during study period.

$b_{\text {Temozolomide was given concurrently with RT for all these patients }}$

${ }^{c}$ Carboplatin $(n=3), \operatorname{CNU}(n=2)$, irinotecan $(n=1)$, veliparib $(n=1)$, buparsilib $(n=1)$.

$d_{\text {Oncolytic retrovirus clinical trial. }}$

encolytic retrovirus clinical trial $(\mathrm{n}=5)$, tumor antigen vaccine clinical trial $(\mathrm{n}=3)$, dendritic cell vaccine $(\mathrm{n}=2)$. Patients in this category also received CCNU ( $n=4)$, carboplatin ( $\mathrm{n}=3)$, nilotinib $(\mathrm{n}=2)$, capecitabine $(\mathrm{n}=1)$, everolimus $(\mathrm{n}=1)$, palbociclib $(\mathrm{n}=1)$, galunisertib $(\mathrm{n}=1)$, and irinotecan $(n=1)$.

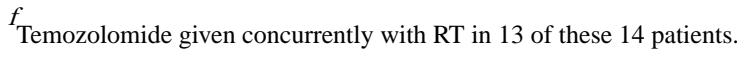

$g_{\text {Carboplatin }(n=9), C C N U}(n=9)$, irinotecan $(n=4)$, erlotinib $(n=2)$, nilotinib $(n=2)$, galunisertib $(n=2)$, thalidomide $(n=1)$, etoposide $(n=1)$, pemetrexed $(n=1)$, lapatinib $(n=1)$, rapamycin $(n=1)$, vemurafenib $(n=1)$, trametinib $(n=1)$, debrafinib $(n=1)$, mipsagargin $(n=1)$, palbociclib $(n=1)$. 
Table 2.

Linear mixed-effects model of amygdala volume change with radiation dose as the main predictor variable.

\begin{tabular}{|l|l|l|l|}
\hline Predictor & Volume change & Standard error & $\boldsymbol{p}$-value \\
\hline Radiation dose & $-0.17 \% / \mathrm{Gy}$ & 0.07 & 0.008 \\
\hline
\end{tabular}

Percent volume change from baseline, 1 year after start of RT, per Gy radiation dose. 